\title{
Standartization of Broth Microdilution Method for Mycobacterium tuberculosis
}

\section{Clarice Queico Fujimura Leite/ ${ }^{+}$, Ana Laura Remédio Zeni Beretta, Ivone Shizuko Anno, Maria Alice da Silva Telles*}

\author{
Faculdade de Ciências Farmacêuticas, Unesp, Caixa Postal 502, 14801-902 Araraquara, SP, Brasil \\ *Instituto Adolfo Lutz, São Paulo, SP, Brasil
}

\begin{abstract}
Indirect drug susceptibility tests of Mycobacterium tuberculosis was done to investigate the accuracy and feasibility of a broth microdilution method (BMM) for determining minimal inhibitory concentrations of conventional drugs against $\mathrm{M}$. tuberculosis. Test drugs included isoniazid (H), rifampicin $(R)$, ethambutol $(E)$, streptomycin $(S)$ and pyrazinamide $(Z)$. Fifty isolates of $M$. tuberculosis from patients who had never received drug therapy, and $H_{37} R_{v}$ strain for control, were evaluated in the system. When comparing this method with the gold standard proportional method in LowensteinJensen medium, sensitivity of 100\% for all drugs and specifities of 91, 100, 96, 98 and 85\% were observed respectively for $H, R, E, S$ and $Z$. The BMM was read faster (14-20 days) than the proportional method (20-28 days). The microdilution method evaluated allows the testing of multiple drugs in multiple concentrations. It is easy to perform and does not require special equipment or expensive supplies. In contrast to radiometric method it does not use radioactive material.
\end{abstract}

Key words: broth microdilution method - Mycobacterium tuberculosis - drugs susceptibility test

In spite of 40 years of effective chemoterapy, tuberculosis is the major cause of death in adults among infectious diseases, being responsible for almost three million deaths annualy in the world (Dolan et al. 1993). Nowadays, the number of tuberculosis cases in Brazil, is supposed to be one hundred thousand cases (Brasil 1997).

However, the tuberculosis control program faces new challenges caused by the increase in multidrug-resistant tuberculosis (Fiuza et al. 1996, Kritski et al. 1996). Drug resistance is a limiting factor for the success of chemotherapy of tuberculosis and evaluation of bacteriostatic and bactericidal activities is essential for the assessment of susceptibility to antimycobacterial drugs before treatment (Kantor \& Laslo 1998).

In this study we analyzed the accuracy and feasibility of a broth microdilution method (BMM) using microplates with 96 wells for determining the minimal inhibitory concentrations (MICs) of conventional drugs. The activity of the drugs were tested against Mycobacterium tuberculosis $\mathrm{H}_{37} \mathrm{R}_{\mathrm{v}}$ as control strain and $50 \mathrm{M}$. tuberculosis strains iso-

This work was supported by a CNPq grant.

+Corresponding author. Fax: +55-16-222.0073. E-mail: leite@iq.unesp.br

Received 7 May 1999

Accepted 22 September 1999 lated from patients without any history of previous tuberculosis treatment. The results were compared with those obtained by the proportion method in Lowestein-Jensen (LJ) medium using the same strains. The BMM may be used to determine the MIC of antibacterial agents against all mycobacteria. It is also useful method for screening new agents.

\section{MATERIAL AND METHODS}

Antimycobacterial agents - Test drugs included isoniazid $(\mathrm{H})$, rifampicin $(\mathrm{R})$, ethambutol (E), streptomycin (S) and pyrazinamide (Z), obtained by Sigma. A stock solution was prepared according to CDC (1985) recommendation. The antimicrobial agents were used in the following concentration: H: 0.05 to 3.2 ; R: 0.25 to $16 ; \mathrm{E}: 0.5$ to $32 ; \mathrm{S}: 0.5$ to 32 and Z: 12.5 to $800 \mu \mathrm{g} / \mathrm{ml}$. All drugs were kept as a stock suspension of $1 \%$ in distilled water except for $\mathrm{R}$ that were dissolved in methanol, and stored at $-25^{\circ} \mathrm{C}$.

Culture and growth of mycobacteria - M. tuberculosis $\mathrm{H}_{37} \mathrm{R}_{\mathrm{v}}$ (pattern strain) obtained from the Collection Institut Pasteur (CIP 14210001) and 50 M. tuberculosis strains obtained from Sanatory Hospital Nestor Goulart Reis (SHNGR), Américo Brasiliense, SP, Brazil, isolated from patients who had never received drug therapy, identified in our laboratory until specie by classical method (Brasil 1994), were mantained on LJ medium slants. Culture suspensions were prepared by growing the inoculum in Middlebrook 7H9 broth (Difco) con- 
taining $0.5 \%$ glycerol and $10 \%$ OADC enrichment (Difco) for five days at $37^{\circ} \mathrm{C}$, without shaking. To prepare the suspension for inoculation, the cultures were vortexed, left for $30 \mathrm{sec}$ to allow the setting of heavy particles than diluted the suspension to reach a turbidity that matched the optical density of a 0.5 MacFarland standard. After this, a 100 fold dilution was prepared and used immediately.

MIC plates preparation - The plates used were 96-well microtitre plates with U-shapped wells. The plates were arranged to give 12 rows by eight lanes and these were filled with $0.1 \mathrm{ml}$ amounts of Middlebrook 7H9 medium, supplemented with OADC enrichment. The stock suspentions of drugs were diluted in Middlebrook 7H9 medium ( $\mathrm{pH} 6.8$, except for $\mathrm{Z}$ with $\mathrm{pH}$ 6.0) and seven serial dilution for each drug were prepared and $0.1 \mathrm{ml}$ volumes were dispensed into plates. Plates were stored at $25^{\circ} \mathrm{C}$ until use.

MIC definition - MIC was defined as the lowest concentration that exhibitis no growth by visual reading, and the strains were considered susceptible for each drug, if their MICs were below or equal to the critical concentration.

Inoculation - Each well was inoculated with 5 $\mu \mathrm{l}$ of bacterial suspension (0.5 MacFarland standard). Medium without antimicrobial agents was inoculated with same suspension and with a 100 fold diluted suspension, as a growing control. The plates were sealed, put in plastic bags and incubated at $37^{\circ} \mathrm{C}$ for 28 days in a moisturized incubator. The bacterial growth (turbidity) was examinated 2, 14, 20 and 28 days after incubation. The results were compared with those obtained by the Proportion Method using the critical concentration of drugs (Brasil 1994).

Proportion Method - Each isolate and $\mathrm{H}_{37} \mathrm{R}_{\mathrm{v}}$ were also tested by the standard proportion method in LJ medium (Brasil 1994). Resistance was defined as growth on drug containing tubes greater than $1 \%$ of the growth on drug free control medium for $\mathrm{H}, \mathrm{R}$, $\mathrm{E}$ and $10 \%$ for $\mathrm{Z}$ and $\mathrm{S}$. A single critical drug concentration was used for this method (Brasil 1994).

\section{RESULTS}

Drug susceptibility tests results of $50 \mathrm{M}$. tuberculosis strains are expressed in the Table. MICs were generally read when the organisms had reached good growth in control wells after 14-17 days but the reading was repeated after 20 and 28 days. Thirty-nine strains had no change in MIC between 17 to 28 day of incubation. While 11 strains changed the MICs but not greater than one fold dilution. This method showed that $78 \%$ of strains were sensitive to all drugs, i.e., the MICs value of drugs were bellow the critical concentration for 39 strains. In $11(22 \%)$ of $M$. tuberculosis studied, the MICs were up to critical concentration for one or more drugs, and those strains were considered resistant. In four strains the MICs values were just one fold dilution greater than critical concentration. Proportion method showed that $90 \%$ of them were sensitive to all drugs and only five strains $(10 \%)$ were drug resistant.

When the BMM was compared with the gold standard proportion method by Kappa test (Ligth 1971) it was observed sensitivity of $100 \%$ for all five drugs and specificity also good except for $\mathrm{Z}$ of $85 \%$ (Table).

\section{DISCUSSION}

The M. tuberculosis susceptibility test used in Brazil is the proportion method in LJ medium using a single drug concentration, named critical concentration (Brasil 1994). Because of the technical complexity of the testing procedures, the test is done only at reference tuberculosis laboratories (Brasil 1995). When comparing the tests, the BMM was read faster (14-20 days) than the proportion method (20-28 days) because $M$. tuberculosis grew more rapidly in broth than in solid egg medium. The correlation of results for each drug was good but the BMM detected a greater number of mutant resistant cells (Table). This method detected early in four $(8 \%)$ strains, a population of mutant resistant cells whose MIC were only one fold dilution greater than critical concentration. The proportion method

TABLE

Drug sensibility of 50 Mycobacterium strains by proportion method and broth microdilution method. Value of sensitivity and specificity of both methods

\begin{tabular}{|c|c|c|c|c|c|c|}
\hline \multirow[t]{3}{*}{ Drugs } & \multicolumn{2}{|c|}{ Proportion method } & \multicolumn{2}{|c|}{ Broth microdilution method } & \multicolumn{2}{|c|}{ Values comparative } \\
\hline & \multicolumn{2}{|c|}{ Number of strains } & \multicolumn{2}{|c|}{ Number of strains } & \multicolumn{2}{|c|}{ Percentage $(\%)$} \\
\hline & Sensitive & Resistant & Sensitive & Resistant & Sensitivity & Specificity \\
\hline Isoniazide & 46 & 4 & 42 & 8 & 100 & 91 \\
\hline Rifampicin & 47 & 3 & 47 & 3 & 100 & 100 \\
\hline Ethambutol & 49 & 1 & 47 & 3 & 100 & 96 \\
\hline Streptomycin & 45 & 5 & 44 & 6 & 100 & 98 \\
\hline Pyrazinamide & 46 & 4 & 39 & 11 & 100 & 95 \\
\hline
\end{tabular}


did not detected those mutants because a single concentration of drugs is used. However Wallace Jr et al. (1993) found 100\% agreement for R, E and S when correlating both methods. They used Middlebrook 7H10 Agar medium for proportion method. It may be that the egg medium used at proportion method contributed for the difference and showed less sensitivity. The discrepancy verified for $\mathrm{Z}$ was theorically expected due to $\mathrm{pH}$ difference of media (pH 5.5 for LJ and 6.0 for Broth 7H9). According to Heifets and Good (1994) the Z has a bactericidal effect at $\mathrm{pH} 5.0$ and the critical concentration at $\mathrm{pH} 6.0$ for this drug should be $100 \mu \mathrm{g} / \mathrm{ml}$ rather than $50 \mu \mathrm{g} / \mathrm{ml}$ that we used.

The possibility of determining the MICs for conventional drugs it should be useful for the treatment of tuberculosis due to partial resistant strains. The knowledge of drugs MICs make possible the use of the drug, until near the maximum concentration that the drug can reach in the human body.

The BMM was used for determining drug susceptibility of M. avium (Telles \& Yates 1994, Collins \& Franzblau 1997), of rapidly growing mycobacteria (Swenson et al. 1982) and other slowly growing mycobacteria including $M$. tuberculosis (Wallace Jr et al. 1986). To improve the mycobacterial growing reading, an association of this method with colorimetric techniques was suggested (GomesFlores et al. 1995, Sato et al. 1997, Franzblau et al. 1998).

In conclusion, the method proposed here is reproducible, comparable with standardized proportion method. It allows testing of multiple drugs at multiple concentrations. It is easy to perform and does not require special equipment or expensive supplies. Differently from radiometric methods it does not use radioactive material.

\section{REFERENCES}

Brasil 1994. Ministério da Saúde/FNS/Cenepi/CNPS/ Centro de Referência Prof. Hélio Fraga, Manual da Bacteriologia da Tuberculose, 2nd ed., Rio de Janeiro, 115 pp.

Brasil 1995. Minstério da Saúde/DNPS/CNCT, Manual de Normas para Controle da Tuberculose, 4th ed., Brasília, 43 pp.

Brasil 1997. Ministério da Saúde/Cenepi, Boletim Epidemiologico 2: 9.

Collins LA, Franzblau SG 1997. Microplate Alamar Blue Assay versus BACTEC 460 system for highthroughput screening of compounds against $\mathrm{Myco-}$ bacterium tuberculosis and Mycobaterium avium. $J$ Clin Microbiol 41: 1004-1009.

CDC - Centers for Disease Control 1985. Public Health Mycobacteriology. A Guide for the Level III Labora- tory, US Department of Health and Human Service, Atlanta, GA, p. 159-184.

Dolan PJ, Raviglione MC, Kochi A 1993. Estimates of future global tuberculosis morbidity and mortality. MMWR 42: 961-963.

Fiuza de Melo FA, Afiune JB, Ribeiro LHG, Almeida de Felice EA, Castelo A 1996. Resistência primária do M. tuberculosis num serviço ambulatorial de referência em São Paulo: evolução por três décadas e comparação com outros estudos nacionais. J Pneumol 22: 3-8.

Franzblau SG, Witzig RS, McLaughlin JC, Torres P, Fuentes P, Cook MB, Madio G, Hernandez A, Dezman MT, Quenzer VK, Feerguson RM, Sheen P, Gilman RH 1998. Rapid, low-technology MIC determination with clinical Mycobacterium tuberculosis isolates by using the Microplate Alaman Blue Assay. J Clin Microbiol 32: 362-366.

Gomes-Flores R, Gupta S, Tamez-Guerra R, Meht RT 1995. Determination of MICs for Mycobacterium avium-intracellulare complex in liquid-medium by colorimetric medium. J Clin Microbiol 34: 1232-1234.

Heifets LB, Good RC 1994. Current laboratory method for the diagnosis of tuberculosis. In BR Bloom, $\mathrm{Tu}$ berculosis Pathogenesis, Protection and Control, ASM Press, Washington, p. 85-122.

Kantor IN, Lazlo A 1997. Tuberculosis laboratory procedures for developing countries. In P Gangadharan \& PA Jenkins (eds), Mycobacteria I Basic Aspeccts, Chapman \& Hall, New York, p. 351-399.

Kritski AL, Marques MJ, Rabahi MF, Vieira MA, Weneck Barroso E, Carvalho CE, Andrade G de N, Bravo de Souza K, Andrade LM, Gontijo PP, Riley LW 1996. Tansmission of tuberculosis to close contacts of patients with multidrug-resistant tuberculosis. Am J Resp Crit Care Med 153: 331-335.

Light RJ 1971. Measures of response agreement for qualitative data: some generalization and alternatives. Psychol Bull 76: 365-377.

Sato DN, Arly DCG, Candido RH, Oliveira DA, Pereira DG, Souza AO, Duran N 1977. Determination of the minimal inhibitory concentration (MIC) and the minimal bactericidal concentration (MBC) of $\mathrm{N}, \mathrm{N}$, dimethyl-2-propen-1-amine derivatives against $M$. tuberculosis. Boll Chim Farm 136: 159.

Swenson JM, Thornsberry C, Silcos VA 1982. Rapidly growing mycobacteria: testing of susceptibility to 34 patients. Antimicrob Agents Chemoter 22: 186-192.

Telles MAS, Yates MD 1994. Simple and double drug susceptibility testing of Mycobacterium avium complex and mycobacteria other than the tubercle (MDTT) bacilli by a micro-dilution both medium minimum inibitory concentration (MIC) method. Tuberc Lung Dis 75: 286-290.

Wallace Jr RJ, Nash DR, Stole LC, Steigrube V 1986. Susceptibility testing of slowly growing Mycobacteria by microdilution, MIC method with 7H9 Broth. J Clin Microbiol 24: 976-981. 